\title{
FACULTADES DE LA POLICÍA JUDICIAL EN LA LUCHA CONTRA LA CORRUPCIÓN EN LOS ESTADOS UNIDOS*
}

\author{
Stephen C. Thaman
}

\section{Resumen}

Este trabajo se ocupa sobre las técnicas de investigación empleadas para luchar contra la corrupción en los Estados Unidos; por supuesto, también es importante abordar las leyes sustantivas utilizadas por la fiscalía para cumplir esa tarea, las prácticas de resolución consensual de estos conflictos a la luz de las súplicas negociadas (plea bargainig) y el papel que cumple el jurado de conciencia en estos casos.

\section{Palabras claves}

Plea Bargaining, Estados Unidos, corrupción, fiscalía, jurado de conciencia, técnicas de investigación.

\begin{abstract}
This work deals with the investigation techniques used to fight corruption in the United States; of course, it is also important to broach the substantive laws used by the Office of the Prosecutor to fulfill that task, the consensual resolution practices of these conflicts in the light of the plea bargaining and the role played by the jury of conscience in these cases.
\end{abstract}

\section{Keywords}

Plea bargaining, United States, corruption, prosecution, jury of conscience, investigation techniques.

\footnotetext{
* Texto de la ponencia presentada en II Congreso Internacional de Policía Judicial Nuevos Retos", organizado por el Instituto de Criminalística, Escuela Mayor de Derecho de la Universidad Sergio Arboleda, Bogotá, el día 20 de octubre de 2017.

** Estudios de Derecho y Doctor en Derecho de la University of California; Center for International and Comparative Law, Saint Louis University; E-mail: thamansc@slu.edu.
} 


\section{Introducción}

Aunque quiero concentrarme en esta jornada en una explicación sobre las técnicas de investigación en los casos de corrupción en los Estados Unidos, es también importante conocer cuáles son las leyes sustantivas utilizadas por la fiscalía para combatir la corrupción, las prácticas de resolución consensual de estos casos en relación con personas físicas y jurídicas y el papel del jurado de conciencia estadounidense en la resolución de estos casos. En el enjuiciamiento de casos de corrupción en los Estados Unidos la Fiscalía federal utiliza, a menudo, leyes muy importantes. Por ejemplo, la Federal Official Bribery and Gratuity Statute (Estatuto Federal de Sobornos y Gratificaciones) (18 U.S.C. § 201) que castiga cohechos y dádivas en relación con funcionarios. Esta Ley fue utilizada, por ejemplo, en el caso notorio de Abscam en virtud del cual durante los años 1977 a 1980 senadores y representantes del Congreso Federal aceptaron cohechos de parte de un agente encubierto, que aparentaba ser un jeque árabe, y que fue el tema de la reciente película American Hustle (Kinskey, 2014, p. 233). Importante es también la Hobbs Act (Ley de Hobbs) (18 U.S.C. § 1951), que castiga la interferencia con el comercio interestatal, y la Foreign Corrupt Practices Act (Ley de prácticas corruptas en el extranjero) (15 U.S.C. $\S \S 78 \mathrm{dd}-1$ a 78 y ss.) que castiga las tentativas de negociantes estadounidenses de pagar cohechos en ultramar.

Se utiliza también la Mail and Wire Fraud (Ley de Fraude por Correo y por Cable) (18 U.S.C. $\S \S 1341,1343)$, en materia de delitos articulados solamente para asegurar la jurisdicción federal, es decir, alegando la utilización de los correos o medios de telecomunicación; así mismo, la Travel Act (18 U.S.C. § 1952) (Interstate and foreign travel or transportation in aid of racketeering enterprises: Ley de Viajes o transportes interestatales y extranjeros para ayudar a empresas del crimen organizado) prohíbe atravesar una frontera interestatal con la intención de cometer ciertos delitos; se ha utilizado a menudo en relación con los cohechos comerciales pero también, como sucedió en el año 2000, contra funcionarios del International Boxing Federation y los miembros del Comité Olímpico en 2003 en relación con los casos de corrupción vinculados con los Juegos Olímpicos de Invierno de Salt Lake City. Finalmente, de importancia enorme es la Racketeer-Influenced and Corrupt Organizations (RICO) (Ley de Chantaje Civil, Influencia y Organizaciones Corruptas) (18 U.S.C. §§ 1961-1968), en la cual se señala en algunos de sus apartes:

(a) Será ilegal para cualquier persona que haya recibido cualquier ingreso derivado, directa o indirectamente, de un patrón de actividad de crimen organizado o mediante el cobro de una deuda ilegal...para usar o invertir, directa o indirectamente, cualquier parte de dicho ingreso, o el producto de tal ingreso, en la adquisición de cualquier interés en, o el establecimiento u operación de cualquier empresa que esté involucrada en, o las actividades de las cuales se afectan el comercio interestatal o extranjero.

(c) Será ilegal para cualquier persona, empleada o asociada con cualquier empresa dedicada o cuyas actividades afecten el comercio interestatal o extranjero, conducir o participar, directa o indirectamente, en la conducción de los asuntos de dicha em- 
presa a través de un patrón de actividad de crimen organizado o de cobro de deudas ilegales.

(d) Será ilegal que una persona conspire para violar cualquiera de las disposiciones de la subsección (a), (b) o (c) de esta sección (18 U.S.C. § 1962 [a, c, d]).

Aunque la jurisdicción natural para enjuiciar los delitos de corrupción debe ser el jurado, es decir, un grupo de doce ciudadanos que delibera sobre los hechos y la culpabilidad, separado del juez técnico, en la realidad más del $97 \%$ de los delitos federales se resuelven con conformidades, sin juicio oral, como fruto de un plea bargain. Por ello, en el ámbito de los casos de corrupción se utilizan también dos procedimientos consensuales que evitan el juicio, pero con un sobreseimiento de la acusación; hablo del deferred prosecution agreements (DPA) (acuerdos de sobreseimiento condicional) y del non-prosecution agreements (NPA) (acuerdos de no acusar). El sobreseimiento condicional (diversion, deferred prosecution) fue introducido originalmente en los tribunales de menores o para resolver delitos de menor importancia, pero se utiliza ahora a menudo en relación con la criminalidad de cuello blanco; se trata de la suspensión de una acusación ya presentada durante un determinado tiempo. En cuanto a los non-prosecution agreements, debe decirse que se trata de la imposición de condiciones sin la presentación de una acusación (Reilly, 2014, 347-406).

Hay tres elementos comunes entre el DPA y el NPA: primero, la admisión de los hechos sirve como confesión si el acusado o sospechoso rompe el acuerdo y hay que celebrar un juicio oral; segundo, se debe cooperar, pues la empresa debe entregar documentos, renunciar a la confidencialidad entre abogado y cliente y, en materia de prescripción, ello supone suspensiones de tres a cinco años. Y, tercero, las empresas, acusadas como personas jurídicas, deben entrar en programas corporativos de cumplimiento (corporate compliance programs).

\section{La importancia de la Cuarta Enmienda en la formulación de estrategias para investigar la corrupción}

La Cuarta Enmienda, promulgada en 1791, señala lo siguiente:

El derecho de los habitantes a que sus personas, domicilios, papeles y efectos se hallen a salvo de pesquisas y aprehensiones arbitrarias, será inviolable, y no se expedirán al efecto mandamientos que no se apoyen en un motivo verosímil, estén corroborados mediante juramento o protesta y describan con particularidad el lugar que deba ser registrado y las personas o cosas que han de ser detenidas o embargadas.

Las cortes interpretan la Cuarta Enmienda cuando articulan las limitaciones de los órganos de la administración de la justicia en la lucha con contra la corrupción, como en la investigación de todos los delitos; hasta el año 1967, el Estado no tenía el poder de secuestrar la propiedad privada de los ciudadanos, si no era un instrumento para cometer un delito, un fruto de un delito, o contrabando. Una escucha no violaba la Cuarta Enmienda si la policía no realizaba un allanamiento de la morada durante la diligencia (Olmstead v. United States, 277 U.S. 438, 1928); 
así mismo, un agente o informante encubridor no violaba la Cuarta Enmienda si era invitado voluntariamente a un domicilio (On Lee v. United States, 343 U.S. 747, 1952). Pero en 1967, en la decisión clave del caso United States v. Katz (389 U.S. 347, 1967), el Tribunal Supremo declaró que "la cuarta enmienda protege la vida privada y no la propiedad" y señaló que "una escucha será legal con autorización judicial y causa probable (probable cause)"; ese mismo año, el Tribunal Supremo declaró que con autorización judicial y causa probable, la policía puede secuestrar cosas privadas no vinculadas con la ilegalidad a título de mera evidencia (mere evidence) (Warden v. Hayden, 387 U.S. 294, 301-302, 1967).

En el caso United States v. Katz, el Tribunal Supremo explicó que la Cuarta Enmienda protege a personas y no a lugares: "Lo que una persona a sabiendas revela al público, aún en su propia casa o despacho, no amerita la protección de la Cuarta Enmienda, mientras que lo que intente mantener de forma confidencial aún en un lugar con acceso público, puede ser constitucionalmente protegido". Aquí hay un aspecto subjetivo: la persona debe exhibir una posibilidad de que la actividad desplegada es privada; y también, un aspecto objetivo: la expectativa debe ser una que la sociedad acepte como "razonable" o fundada.

En su jurisprudencia posterior después de Katz, el Tribunal Supremo reconoció limitaciones a la tutela de la Cuarta Enmienda; por ejemplo, el pueblo no tendría una "fundada expectativa de privacidad en información comunicada a un tercero, si una persona invita a un agente o informante encubierto a su esfera privada y confía en él", en este caso "el agente puede registrar la conversación y transmitirla simultáneamente a la policía” (United States v. White, 401 U.S. 745, 1971).

Así las cosas, cuando una persona le da acceso a información a un tercero, ella asume el riesgo en relación con su revelación. En el mismo sentido, la información comunicada a un proveedor de servicios como los números de teléfono marcados por una persona que llama (Smith v. Maryland, 442 U.S. 735, 1979), o la información sobre transacciones financieras a un banco (California Bankers Ass'n v. Shultz, 416 U.S. 21, 1974; United States v. Miller, 425 U.S. 435, 1976) carecen de protección en la Cuarta Enmienda. Naturalmente, si alguien abandona algo, por ejemplo, en la basura, perdería la tutela de la Cuarta Enmienda sobre la cosa (Califonia v. Greenwood, 486 U.S. 35, 1988); pero se debe recordar que el Tribunal Supremo señala solo la protección mínima exigida por la Constitución. Los Estados, sin embargo, pueden interpretar sus propias constituciones y leyes para otorgar más protección en una esfera o en otra; por ejemplo, los Estados de Oregón (State v. Galloway, 109 P.3d 383, Or. App. 2005), Vermont (State v. Morris, 680 A.2d 90, Vt. 1998), New México (State v. Crane, 254 P.3d 117, N.M. App. 2011) y New Hampshire (State v. Goss, 834 A.2d 216, N.H. 2003) prohíben el registro de basura que un ciudadano entrega al depósito de desperdicios en una bolsa o en un contenedor opaco.

Así mismo, muy importantes son las decisiones que deniegan la protección para la conducta en espacios públicos o en campo abierto. Por ejemplo, el Tribunal 
Supremo ha permitido el uso de localizadores electrónicos (beepers) para seguir y vigilar a personas sospechosas en la esfera pública (United States v. Knotts, 460 U.S. 276, 1983), pero no si el encargado de la vigilancia se entromete en un lugar privado como un domicilio (United States v. Karo, 468 U.S. 705, 1984). Pero en la decisión del caso Jones v. United States (565 U.S. 400, 2012), la mayoría del Tribunal Supremo decidió que la colocación secreta de un aparato GPS en un vehículo constituye una violación del derecho de propiedad sobre el automotor, pero opiniones separadas de dos Magistrados han mantenido la idea de que el uso por un tiempo prolongado de un localizador GPS viola la expectativa de privacidad (privacy) protegida por la Cuarta enmienda. Así mismo, los Estados de South Dakota (State v. Zahn, 812 N.W.2d 490, S.D. 2012), Wisconsin (State v. Brereton, 826 N.W.2d 369, Wis. 2013), Massachusetts (Commonwealth v. Rousseau, 990 N.E.2d 543, Mass. 2013), New York (People v. Weaver, 909 N.E.2d 1195, N.Y. 2009) y Washington (State v. Jackson, 76 P.3d 217, Wash. 2003), exigen un auto judicial con una probable causa (probable cause) con vigilancia a largo plazo.

\section{Las leyes que regulan la interceptación de comunicaciones y el acceso a la información electrónica archivada}

Las leyes claves en los Estados Unidos para regular la interceptación de comunicaciones son la Ley de Escuchas, llamada Título Tercero (Title III), de 1968, que se utiliza para la investigación de casos criminales de índole típico (18 U.S.C. §§ 2510-20) y la Ley de Vigilancia de Inteligencia Extranjera de 1978 (Foreign Intelligence Surveillance Act-FISA), que se ocupa de la vigilancia de agentes extranjeros en casos de seguridad nacional, espionaje y terrorismo internacional (50 U.S.C. §§ 1801 et seq.). Las otras leyes claves son la Ley de Comunicaciones almacenadas de 1986 (Stored Communications Act), que regula el acceso a comunicaciones archivadas (correos electrónicos, etc.), y la Ley de Privacidad de comunicaciones electrónicas (Electronic Communications Privacy Act) también de 1986, que regula el uso de teléfonos celulares (móviles) y comunicación por internet. Finalmente, la Cuarta Enmienda establece los principios residuales cuando no se aplican las leyes arriba citadas, por ejemplo, el uso de agentes encubiertos, etc.

Title III (La Ley de Escuchas) fue promulgada después de la decisión de Katz. Es una ley orgánica aplicable a todo los Estados (como estándar mínimo) y exige una protección más amplia que la Cuarta enmienda propia. Por ejemplo, Title III tiene una regla de exclusión (prueba prohibida) más estricta que la de la Cuarta Enmien$\mathrm{da}$, sin excepciones para la "buena fe" y con aplicación en procedimientos civiles y administrativos, etc. Hay también una exigencia de necesidad: la de explicar por qué medidas menos lesivas no fueron utilizadas.

Pero La Ley de Escuchas no se aplica a los metadatos de telecomunicaciones, es decir, los números de teléfono marcados. Tampoco a las comunicaciones archivadas por los proveedores o en mensaje de voz, ni en la ubicación de un teléfono móvil. Por eso, el fiscal no necesita autorización judicial basado en probable cause, para acceder a esta información, sino solo un subpoena (citación), que exige solamente la 
afirmación de que la información será de uso en la investigación. Y no habrá una regla de exclusión si la fiscalía o la policía ignoran las exigencias de la ley.

\section{Las tácticas investigativas en la lucha contra la corrupción}

Naturalmente, las investigaciones de corrupción tienen que identificar la actividad sospechosa (véase Bernstein, $\mathrm{s} / \mathrm{f}$ ); por eso, utilizan pesquisas tanto reactivas o históricas como proactivas y secretas. Para identificar sospechosos potenciales, el F.B.I. utiliza fuentes policiales y gubernamentales, las quejas de ciudadanos, o indaga a negociantes, políticos y empleados infelices. También, emplean informantes pagados y a acusados que cooperan con la policía para aliviar las consecuencias de su propia conducta delincuente; en fin, sacan también pistas de investigación en la prensa, internet o en otros medios de telecomunicación.

Además, tienen a su alcance bases de datos comerciales como los de Choicepoint y otra información crediticia y también contribuciones políticas. Tienen acceso a un banco de datos sobre transacciones financieras sospechosas, que los bancos deben recoger según la Bank Secrecy Act (FinCen Form 112). En fin, utilizan información revelada en casos civiles o administrativos. Incluso, el fiscal puede utilizar mandamientos emitidos por el Gran Jurado (grand jury), un órgano inquisitorio compuesto de laicos bajo la dirección del fiscal, para exigir la entrega de documentos importantes en la custodia de instituciones financieros, proveedores de telecomunicaciones, y sociedades y empresas. También la fiscalía, con una citación del Gran Jurado, puede obligar a un testigo a declarar, un poder que el fiscal o la policía no tienen en los Estados Unidos. Naturalmente, ello solo se hace cuando el testigo no quiere hacer una declaración ante el fiscal en forma voluntaria.

En investigaciones reactivas hay varias diligencias al alcance de la fiscalía durante una investigación en casos de corrupción. Entre ellas, están, por ejemplo, la revisión de mail covers. Al respecto, debe decirse que desde hace mucho tiempo el Departamento de Correos hace fotografías de cada paquete o carta enviada; como los números de teléfono, la dirección es información expuesta al público y no tiene protección de la Cuarta Enmienda. Además, se puede hacer una inspección de la basura de un sospechoso; cuando una persona bajo investigación se pone nerviosa, a menudo tira documentos importantes en la basura. Como ya se ha dicho, no se exige autorización judicial para estas medidas.

También el fiscal con autorización judicial puede otorgar a un testigo inmunidad para obligarlo a declarar contra sus intereses; sin embargo, los fiscales no lo hacen a menudo porque los jurados no confían siempre en testigos que declaran bajo inmunidad. El fiscal puede también conseguir la cooperación de un co-imputado después de haber concluido un acuerdo de cooperación, una especie de plea bargain, en la que un acusado llega a un acuerdo sobre su propio caso. Los fiscales piensan que los jurados suelen creer más a un testigo que declara en un juicio de un cómplice con una promesa implícita de conseguir una pena reducida, que ha admitido su culpabilidad, que a uno bajo inmunidad que no confronta ninguna 
pena. Yo no comparto esta opinión. Según mi juicio, ambos testigos carecen de credibilidad.

Además, agréguese que son muy importantes las citaciones de información archivada sobre la actividad telecomunicativa de un sospechoso; para obtener la información sobre la actividad archivada se exige solamente una citación sin necesidad de una causa probable (probable cause), pero se exige un auto judicial para conseguir el contenido de las conversaciones. A veces el investigador quiere determinar la identificación de un usuario de un teléfono, de un ordenador o de una cuenta electrónica.

Por ello, en cualquier mandamiento judicial para registrar una oficina o una empresa se incluye el poder de secuestrar ordenadores y de registrar las informaciones digitales archivadas dentro; es, pues, usual que especialistas en el área de ordenadores acompañen a la policía durante la ejecución del mandamiento, para facilitar la copia del contenido de los ordenadores en el sitio y no sea necesario transportar el aparato y hacerlo más tarde.

Entre las medidas al alcance de la fiscalía en investigaciones proactivas se debe mencionar la vigilancia por un tiempo extendido; para ello, no se exige autorización judicial en el sistema federal, pero desde la decisión en el caso Jones (Jones v. United States, 565 U.S. 400, 2012), no se puede fijar un localizador-GPS al vehículo bajo vigilancia sin mandato judicial. También, se puede hacer una interceptación coetánea a la actividad telefónica, es decir, de los megadatos de comunicación telefónica, sin causa probable y mediante mandato judicial, porque la Cuarta Enmienda no protege esa información. Con un mandato judicial bajo el Título III, la fiscalía puede implantar un micrófono en un domicilio para escuchar y grabar de forma acústica las conversaciones entre los presentes; desde luego, un auto de escuchas exige causa probable (probable cause) y el Título III (Title III) impone deberes de minimizar la intercepción de conversaciones ajenas y de informar al juez que emitió el auto, sobre los contenidos de las conversaciones interceptadas. Pero, en verdad, se utiliza raramente en casos de corrupción; por ejemplo, en 2014 y de parte de la Fiscalía del Estado de Pennsylvania se autorizaron 3.554 escuchas de las cuales 3.170 lo fueron para la droga, 30 para conspiración, 72 para la Ley RICO y solo 16 para casos de corrupción.

De igual forma, se pueden grabar conversaciones de un sospechoso sin el mandato exigido por el Título III, si la policía consigue el acuerdo de un participante en la conversación; por ejemplo, en la investigación por los eventos de corrupción de la FIFA, el F.B.I. obtuvo la participación de Chuck Blazer para grabar conversaciones incriminatorias con otros funcionarios de ese organismo. De la misma manera, esto fue muy importante en la investigación y el enjuiciamiento de los sospechosos del caso Abscam. Ahora bien, según la jurisprudencia del Tribunal Supremo no se exige un mandato judicial para enviar a un agente o informante encubierto a un domicilio a grabar clandestinamente conversaciones al interior si el agente consigue una invitación del sospechoso (véase la decisión de White); sin 
embargo, por lo menos seis Estados exigen autorización judicial para enviar a un agente encubierto o informante a un domicilio para hacer grabaciones clandestinas (People v. Beavers, 227 N.W.2d 511, Mich. 1975; State v. Glass, 583 P.2d 872, Alaska 1978; Commonwealth v. Brion, 652 A.2d 287, Pa. 1994; State v. Bridges, 925 P.2d 357, Haw. 1996; State v. Geraw, 795 A.2d 1219, Vt. 2002; State v. Mullens, 650 S.E.2d 169, W.Va. 2007).

También se utilizan agentes encubiertos para tratar de inducir al sospechoso a admitir un delito ya cometido, que naturalmente afecta el derecho a no autoincriminarse. Sin embargo, según la jurisprudencia del Tribunal Supremo, no viola la Quinta Enmienda que tutela el derecho a no testificar contra si el hecho de utilizar agentes encubiertos en las cárceles para inducir admisiones de culpabilidad, por lo menos antes de que el fiscal emita una acusación formal (Illinois v. Perkins [1990]. 496 U.S. 292). Después de una acusación formal, un agente encubierto puede ocupar la celda junto a un acusado, pero no lo interroga activamente (United States v. Henry [1980]. 477 U.S. 264; Kuhlmann v. Wilson [1986], 477 U.S. 436).

A lo anterior, añádase que el uso de agentes encubiertos ha sido muy importante para el descubrimiento y enjuiciamiento de casos de corrupción; por eso, los agentes federales tratan siempre de utilizar agentes encubiertos cuando sospechan que se encuentran ante una situación de corrupción. Ellos prefieren funcionarios encubiertos en vez de informantes o acusados cooperantes, porque el jurado suele confiar en los agentes y no necesariamente en los informantes, sobre todo cuando estos son pagados. Los agentes encubiertos tratan de grabar todas las conversaciones bien sea de forma consensuada o bien con una escucha a la luz del Título III. Para los fiscales, las grabaciones son irrecusables y los jurados confían en ellas.

La llamada "bala de plata," u objetivo quintaescencial de las fuerzas de seguridad en la investigación de casos de corrupción es el flip o capirotazo de un participante en la organización corrupta, para que éste termine cooperando con la investigación. El F.B.I. ha investigado a la FIFA por conspiración de violación del RICO, fraude, violación del travel act, etc. y ha realizado el capirotazo de Chuck Blazer, un ex-funcionario ejecutivo de FIFA. Blazer fue investigado por no haber pagado impuestos por millones de dólares de ingresos secretos, según el New York Post, y comenzó a cooperar con el FBI en la investigación de la FIFA. Secretamente, según cabe presumir, él grabó con un aparato las conversaciones con sus colegas durante los juegos olímpicos en Londres durante el 2012, donde la gran mayoría de las figuras más importantes de la FIFA se encontraban (Gibson, 2014).

\section{El atrapamiento (entrapment)}

Bastante polémica es la organización de una trampa (sting) para provocar la comisión de un delito, por ejemplo, la aceptación de un cohecho; esta táctica para perseguir la delincuencia genera dificultades con los llamados delitos provocados y problemas con el respeto del debido proceso. En el sistema federal, un agente especial a cargo del F.B.I. (llamado special agent in charge) puede autorizar cualquiera 
actividad realizada por parte de un agente encubierto si piensa que es proporcional en relación con la gravedad del delito objeto de investigación; esta autorización puede durar hasta seis meses que pueden llegar a ser duplicados. Si la operación implica una cantidad de dinero grande, la vigilancia de funcionarios, la entrega de drogas, el pagamento de sobornos, la infiltración de organizaciones terroristas, o la interacción con abogados, sacerdotes o médicos, etc. se exige la autorización del cuartel general del F.B.I., pero estas prohibiciones son solo internas y su violación no tiene importancia legal alguna.

En los Estados Unidos el atrapamiento, es decir, la incitación de parte de agentes oficiales a la comisión de un delito es una excusa que desencadena un veredicto de no culpabilidad si la acepta el jurado o el juez, según la teoría de la jurisdicción. Los tribunales federales y la mayoría de tribunales estatales reconocen una teoría subjetiva en materia del atrapamiento; según la decisión del Tribunal Supremo del año 1932 (Sorrells v. United States, 287 U.S. 435, 1932) : “Cuando la intención criminal surge con los funcionarios del gobierno y ellos inculcan en la mente de un inocente la disposición a cometer el delito para poder enjuiciarlo, eso es atrapamiento (entrapment)"; los agentes pueden dar a una persona la oportunidad o los instrumentos para cometer el delito. Desde luego, la línea importante es aquella que se presenta entre una trampa para un incauto inocente y una trampa para un incauto culpable.

Si el acusado tenía una predisposición para cometer el delito, el será culpable aun cuando la policía utilice incentivos o tácticas coercitivas que podrían haber provocado el delito de parte de un inocente; de ahí que la teoría subjetiva deje al jurado la tarea de decidir la culpabilidad y la cuestión atinente a la predisposición y, añádase, el fiscal puede introducir información sobre antecedentes penales para convencerlo de la predisposición correspondiente. Ahora bien, según la teoría objetiva si la conducta de la policía hubiera provocado la comisión del delito por parte de un inocente, es irrelevante el hecho de que hubiese existido la predisposición por parte del acusado; por ello, según esta construcción académica el juez determina si la conducta policial hubiera podido inducir a un inocente a cometer el delito. § 2.13 del Código Modelo de 1962 refleja la teoría objetiva, y unos 10-15 Estados, entre ellos California, han adoptado esta teoría minoritaria.

Algunos estados reconocen también una violación del debido proceso en casos de conducta ultrajosa por parte de la policía (outrageous police conduct) que es semejante a la teoría objetiva (United States v. Twigg, 588 F.2d 373, (3d Cir. 1978; People v. Isaacson, 406 N.Y.S.2d 714, N.Y. 1978; Nadeau v. State, 683 So.2d 504, Fla.App. 1995; State v. Lively, 921 P.2d 1035, Wash. 1996; Commonwealth v. Monteagudo, 693 N.E.2d 1381, Mass. 1998; People v. Ming, 738 N.E.2d 628, Ill.App. 2000); los acusados en los famosos casos del "Abscam" han alegado la figura del atrapamiento como argumento en pro de su defensa; uno de ellos ha convencido al juez de instancia de que fue la víctima de ello (entrapment y outrageous police conduct) pero no tuvo éxito ante el Tribunal de Apelación. 


\section{El triunfo de plea bargaining}

Todos de los acusados en los casos de Abscam fueron condenados por jurados, que han rechazado la alegación del atrapamiento y, como se ha mencionado, casi no hay juicios con jurado cuando se trata de la acusación de una persona jurídica para violaciones del FCPA. De verdad, casi todos los acusados, sobre todo en el sistema federal, renuncian a su derecho a ser enjuiciados por un jurado a favor de una conformidad o guilty plea. En mi opinión, el sistema estadounidense del plea bargaining es inherentemente coercitivo. En el caso de Bordenkircher v. Hayes (434 U.S. 357, 1978), el Tribunal Supremo ha proclamado que la oferta de imponer una pena privativa de libertad de cinco años si el acusado confiesa a cambio de una cadena perpetua en caso de condena, no violaba el debido proceso. Aunque las pautas para la aplicación de la pena (las U.S. Sentencing Guidelines) prevén un descuento de una tercera parte con un guilty plea, el descuento es en realidad de dos terceras partes de la pena. Después de un guilty plea, la pena promedio imponible en los tribunales federales es 54.7 meses de prisión, mientras que la pena media después de un juicio oral es 153.7 meses (Turner, 2005, p. 199).

\section{Las estadísticas}

Entre 1976, cuando el Ministerio de Justicia federal empezó de compilar estadísticas, y 2013, los Estados con la cantidad más grande de condenas para corrupción eran: (1) New York, con 2.657; (2) California, con 2.549; y, (3) Illinois, con 1.982 (1.642 de ellas en los alrededores de Chicago) (Simpson et al., 2015. p. 4); en el año 2013, hubo 337 enjuiciamientos en relación con funcionarios federales. De ellos terminaron 315 con condenas y 113 casos quedaron pendientes; hubo, además, 133 casos en relación con funcionarios estatales con 119 condenas y 68 quedaron pendientes. Así mismo, se presentaron 334 casos en relación con funcionarios municipales, con 103 condenas y 149 que quedaron pendientes; de igual manera, hubo otros 330 otros casos con 300 condenas y 169 quedaron pendientes. En suma, se presentaron 1.134 casos con 1037 condenas, y 499 casos quedaron pendientes.

\section{Referencias}

Bernstein, D. Special Investigative Techniques in Public Corruption Investigations. Recuperado de https://thesiliconcoup.weebly.com/uploa ds /1/3/3/9/13392840/07_30_2008_track_c_0400_0450_special_investigative_ techniques_in_public_corruption_investigations.pdf

Caso Olmstead v. United States, 277 U.S. 438 (1928). Recuperado de https:// supreme.justia.com/cases/federal/us/277/438/ case.html

Caso Sorrells v. United States, 287 U.S. 435 (1932). Recuperado de https: / / supreme. justia.com/cases / federal/us/287/435/case.html

Caso On Lee v. United States, 343 U.S. 747 (1952). Recuperado de https: / / supreme. justia.com/cases / federal/us/343/747/ 
Caso United States v. Katz 389 U.S. 347 (1967). Recuperado de https: / / supreme. justia.com/cases/federal/us/389/347/ case.html

Caso Warden v. Hayden, 387 U.S. 294, 301-302 (1967). Recuperado de https:// supreme.justia.com/cases / federal/us/387/294/case.html

Caso United States v. White, 401 U.S. 745 (1971). Recuperado de https: / / supreme. justia.com/cases / federal/us/401/745/case.html

Caso California Bankers Ass'n v. Shultz, 416 U.S. 21 (1974). Recuperado de https: / / www.casemine.com/judgement/us/5914c6b4add7b049347dd175

Caso People v. Beavers, 227 N.W.2d 511 (Mich. 1975). Recuperado de https: / law. justia.com/ cases / michigan / supreme-court/1975/54847-2.html

Caso United States v. Miller, 425 U.S. 435 (1976). Recuperado de https: / / supreme. justia.com/cases / federal/us/425/435/

Caso State v. Glass, 583 P.2d 872 (Alaska 1978). Recuperado de https://www. courtlistener.com/opinion/1290844/ state-v-glass /

Caso United States v. Twigg, 588 F.2d 373 (3d Cir. 1978). Recuperado de https:/ / law.resource.org / pub/us / case/ reporter / F2 / 588/588.F2d.373.78-1348.781315.html

Caso People v. Isaacson, 406 N.Y.S.2d 714 (N.Y. 1978). Recuperado de https:// casetext.com/case/ people-v-isaacson

Caso Bordenkircher v. Hayes (434 U.S. 357 (1978). Recuperado de https: / / supreme. justia.com/cases / federal/us/434/357/ case.html

Caso Smith v. Maryland, 442 U.S. 735 (1979). Recuperado de https://supreme. justia.com/cases/federal/us/442/735/case.html

Caso United States v. Henry, 477 U.S. 264 (1980). Recuperado de https: / / supreme. justia.com/cases / federal/us/447/264/

Caso United States v. Knotts, 460 U.S. 276 (1983). Recuperado de https: / / supreme. justia.com/cases/federal/us/460/276/ case.html

Caso United States v. Karo, 468 U.S. 705 (1984). Recuperado de https: / / supreme. justia.com/cases / federal/us/468/705/case.html

Caso Kuhlmann v. Wilson, 477 U.S. 436 (1986). Recuperado de https:/ / supreme. justia.com/cases / federal/us/477/436/

Caso California v. Greenwood, 486 U.S. 35 (1988). Recuperado de https: / / supreme. justia.com/cases/federal/us/486/35/case.html

Caso Illinois v. Perkins, 496 U.S. 292 (1990). Recuperado de https: / / supreme.justia. com/cases/federal/us / 496/292/ case.html 
Caso Commonwealth v. Brion, 652 A.2d 287 (Pa. 1994). Recuperado de https:/ / www.courtlistener.com/opinion/1934626/ com-v-brion/

Caso Nadeau v. State, 683 So.2d 504 (Fla.App. 1995). Recuperado de https:/ / www. courtlistener.com/opinion/1105653/nadeau-v-state/

Caso State v. Bridges, 925 P.2d 357 (Haw. 1996). Recuperado de https: / law.justia. com/ cases / hawaii / supreme-court/1996/16800-2.html

Caso State v. Lively, 921 P.2d 1035 (Wash. 1996). Recuperado de https: / law.justia. com/ cases/washington/ supreme-court/1996/60389-8-1.html

Caso Commonwealth v. Monteagudo, 693 N.E.2d 1381 (Mass. 1998). Recuperado de https:/ / massachusetts.lexroll.com/ commonwealth-v-monteagudo-427mass-484-1998/

Caso State v. Morris, 680 A.2d 90 (Vt. 1998). Recuperado de https://www. courtlistener.com/opinion/1992904/state-v-morris /

Caso People v. Ming, 738 N.E.2d 628 (Ill.App. 2000). Recuperado de https:// caselaw.findlaw.com/il-court-of-appeals/1062512.html

Caso State v. Geraw, 795 A.2d 1219 (Vt. 2002). Recuperado de https://www. courtlistener.com/opinion/1439872/ state-v-geraw /

Caso State v. Jackson, 76 P.3d 217 (Wash. 2003). Recuperado de https:/ / law.justia. com/cases/washington/ supreme-court/2003/72799-6-1.html

Caso State v. Goss, 834 A.2d 216 (N.H. 2003). Recuperado de https://caselaw. findlaw.com/nh-supreme-court/1128324.html

Caso State v. Galloway, 109 P.3d 383 (Or. App. 2005). Recuperado de https: / / www. courtlistener.com/opinion/2520315/ state-v-galloway /

Caso State v. Mullens, 650 S.E.2d 169 (W.Va. 2007). Recuperado de https:/ / www. courtlistener.com/opinion/1331464 / state-v-mullens /

Caso People v. Weaver, 909 N.E.2d 1195 (N.Y. 2009). Recuperado de https:// caselaw.findlaw.com/ny-court-of-appeals / 1140764.html

Caso State v. Crane, 254 P.3d 117 (N.M. App. 2011). https: / / caselaw.findlaw.com/ nm-supreme-court/1671543.html

Caso Jones v. United States, 565 U.S. 400 (2012). Recuperado de https: / / supreme. justia.com/cases / federal/us/565/400/

Caso State v. Zahn, 812 N.W.2d 490 (S.D. 2012). Recuperado de https:/ /www. leagle.com/decision/insdco20120315648

Caso State v. Brereton, 826 N.W.2d 369 (Wis. 2013). Recuperado de: https:/ / www. leagle.com/decision/inwico20130206f59 
Caso Commonwealth v. Rousseau, 990 N.E.2d 543 (Mass. 2013). Recuperado de https: / / casetext.com/ case/ commonwealth-v-rousseau-2

Gibson, O. (2014, noviembre 3). FBI FIFA bugging reports should prompt World Cup investigation, says M.P. The Guardian. Recuperado de http://www. theguardian.com/ football/2014/ nov / 03 / chuck-blazer-fifa-fbi-buggingworld-cup-bidding-investigation

Kinskey, M. W. (2014). American Hustle: Reflections on ABSCAM and the Entrapment Defense, 41 en American Journal of Criminal Law, 41(3), 233-260.

Reilly, P. (2014). Negotiating Bribery: Towards Increased Transparency, Consistency and Fairness in Pretrial Bargaining Under the Foreign Corrupt Practices Act. En Hastings Business Law Journal N. ${ }^{\circ}$ 10:2, 347-406.

Simpson, D., Th. Gradel, M. Mouritsen \& J. Johnson (2015, mayo 28). Chicago: Still the Capital of Corruption, Anti-Corruption Report N. ${ }^{\circ}$ 8, University of Illinois at Chicago Department of Political Science, 1-9. Recuperado de https://pols. uic.edu / docs / default-source / chicago_politics / anti-corruption_reports / corruption-rpt-8_final-052715.pdf?sfvrsn=0

Turner, J. I. (2006). Judicial Participation in Plea Negotiations: A Comparative View. En American Journal of Comparative Law, (Vol. 94), 501-570. 